\title{
Palladium-Catalyzed Direct C-3 Alkynylation of Indolizines with (2,2-Dibromovinyl)arenes
}

\author{
Chuang Shen, ${ }^{\dagger}$ Yuzhu Yang, ${ }^{\dagger}$ Zhanxiang Liu, ${ }^{*}{ }^{\dagger}$ Yuhong Zhang*, ${ }^{\dagger, \ddagger}$ \\ ${ }^{\dagger}$ ZJU-NHU United R\&D Center, Department of Chemistry, Zhejiang University, Hangzhou \\ 310027, China \\ ${ }^{\ddagger}$ State Key Laboratory of Applied Organic Chemistry, Lanzhou University, Lanzhou 730000, \\ China
}

E-mail: liuzhanx@zju.edu.cn, yhzhang@zju.edu.cn, Fax: +86-571-87953244; Tel: +86-571-87952723;

\section{Supporting Information}

\section{Content}

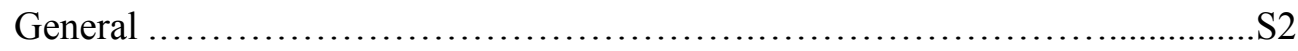

Preparation and Characterization of Starting Materials..................................S2

Typical procedure for the product.......................................... 3

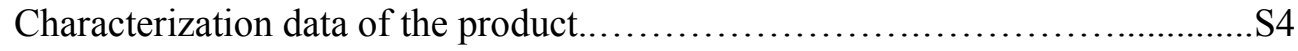

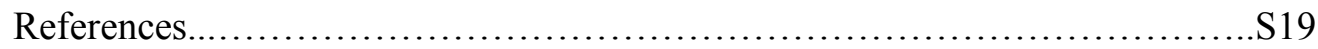


General

Infrared spectra were recorded with a FTIR spectrometer. NMR spectra were recorded for ${ }^{1} \mathrm{H} \mathrm{NMR}$ at $400 \mathrm{MHz}$ or $500 \mathrm{MHz}$, and ${ }^{13} \mathrm{C} \mathrm{NMR}$ at $100 \mathrm{MHz}$ or $125 \mathrm{MHz}$ using TMS as internal standard. The following abbreviations were used to describe peak patterns where appropriate: singlet (s), doublet (d), doublet of doublets (dd), triplet (t), quartet (q), multiplet (m), broad resonances (br). Mass spectroscopy data of the products were collected on an HRMS-EI-TOF instrument or a low-resolution MS instrument using EI or ESI ionization.

Preparation and Characterization of Starting Materials: ${ }^{[1]}$

Step 1: To a solution of pyridines $(100 \mathrm{mmol})$ in EtOAc $(60 \mathrm{~mL})$ was added the appropriate bromoacetic acid $(100 \mathrm{mmol})$. After the mixture was stirred for $3 \mathrm{~h}$ at r.t., the solid was filtered and dried in air to give $\mathrm{N}$-(carboxymethyl)pyridinium bromides as white solid.

Step 2: A suspension of $\mathrm{N}$-(carboxymethyl)pyridinium bromides (10 mmol), alkene (50 mmol), $\mathrm{Et}_{3} \mathrm{~N}(1.5 \mathrm{~mL})$ and $\mathrm{MnO}_{2}(80 \mathrm{mmol})$ in toluene $(80 \mathrm{~mL})$ was stirred at 90 ${ }^{\circ} \mathrm{C}$ for $2 \mathrm{~h}$ (monitored by TLC). After the mixture was cooled to r.t., the solid was filtered off and washed with acetone. The combined filtrates were evaporated to give a residue, which was purified by chromatography (silica gel, ethyl acetate/petrol ether $=1 / 5)$ to give the corresponding indolizines stored in the refrigerator. 
Typical procedure for the product:

A mixture of indolizines $(0.3 \mathrm{mmol})$, vinyl dibromides $(0.9 \mathrm{mmol}), \mathrm{Pd}(\mathrm{OAc})_{2}(7$ mg, $10 \mathrm{~mol} \%$ ), DPEPhos (30 mg, $20 \mathrm{~mol} \%$ ), AgOAc (100 mg, $0.6 \mathrm{mmol})$, CsOAc (170 mg, $0.9 \mathrm{mmol}$ ) in DMF (2 mL) was stirred at $100{ }^{\circ} \mathrm{C}$ under $\mathrm{N}_{2}$ for $24 \mathrm{~h}$. Afterward, the mixture was cooled to room temperature, filtered through a pad of celite. The crude product was dissolved in $\mathrm{Et}_{2} \mathrm{O}(20 \mathrm{~mL})$, washed with water $(2 \times 20$ $\mathrm{mL})$, brine $(20 \mathrm{~mL})$, then dried over $\mathrm{Na}_{2} \mathrm{SO}_{4}$. The solvent was evaporated under reduced pressure, and the residue was subjected to flash column chromatography to obtain the desired product. 
Characterization data of the products

\section{3-(Phenylethynyl)indolizine-1-carbonitrile ( 3a ) ${ }^{[2]}$}

White solid; m.p. $276-278{ }^{\circ} \mathrm{C}$; ${ }^{1} \mathrm{H}$ NMR ( $400 \mathrm{MHz}, \mathrm{CDCl}_{3}$, TMS ) $\delta 8.37$ ( d, $J=6.8$ $\mathrm{Hz}, 1 \mathrm{H}), 7.67$ ( d, $J=8.8 \mathrm{~Hz}, 1 \mathrm{H}$ ), 7.56-7.58 ( m, $2 \mathrm{H}$ ), 7.38-7.39 ( m, $3 \mathrm{H}$ ), 7.28 $(\mathrm{s}, 1 \mathrm{H}), 7.19(\mathrm{t}, J=7.6 \mathrm{~Hz}, 1 \mathrm{H}), 6.92$ ( t, $J=6.4 \mathrm{~Hz}, 1 \mathrm{H}) ;{ }^{13} \mathrm{C} \mathrm{NMR}(100 \mathrm{MHz}$, $\left.\mathrm{CDCl}_{3}\right) \delta 138.0,131.4,128.9,128.5,125.7,124.0,122.1,121.4,117.9,113.8,97.2$, 82.3. HRMS (EI) Calcd for $\mathrm{C}_{17} \mathrm{H}_{10} \mathrm{~N}_{2}\left(\mathrm{M}^{+}\right)$242.0844; Found, 242.0851.
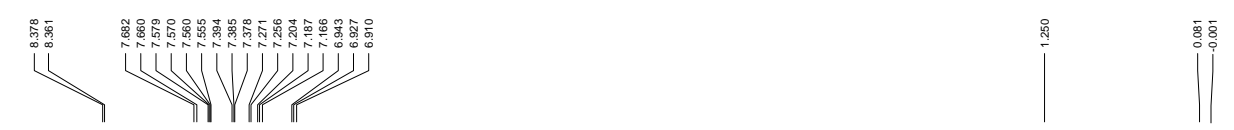

$3 a-\mathrm{H}$
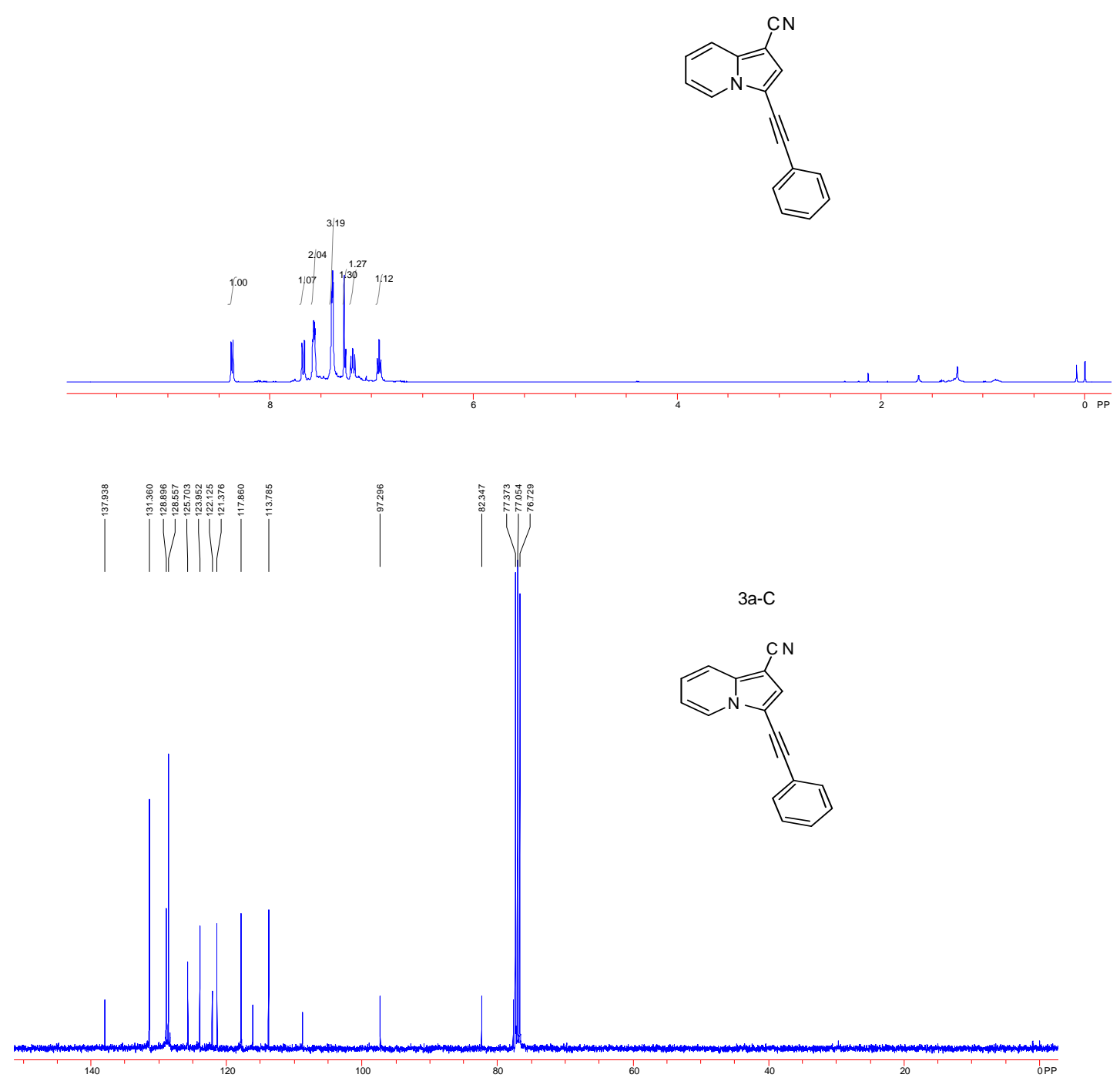


\section{Methyl 3-(phenylethynyl)indolizine-1-carboxylate ( 3b ) ${ }^{\text {[2] }}$}

White solid; m.p. $254-256{ }^{\circ} \mathrm{C} .{ }^{1} \mathrm{H}$ NMR ( $400 \mathrm{MHz}, \mathrm{CDCl}_{3}$, TMS ) $\delta 8.36$ ( d, $J=6.8$ $\mathrm{Hz}, 1 \mathrm{H}$ ), 8.23 ( d, $J=9.2 \mathrm{~Hz}, 1 \mathrm{H}$ ), 7.56-7.58 ( m, $2 \mathrm{H}$ ), 7.52 ( s, $1 \mathrm{H}$ ), 7.37-7.39 $(\mathrm{m}, 3 \mathrm{H}), 7.17(\mathrm{t}, J=8.0 \mathrm{~Hz}, 1 \mathrm{H}), 6.88(\mathrm{t}, J=6.8 \mathrm{~Hz}, 1 \mathrm{H}), 3.91(\mathrm{~s}, 3 \mathrm{H}) ;{ }^{13} \mathrm{C}$ NMR ( $100 \mathrm{MHz}, \mathrm{CDCl}_{3}$ ) $\delta 164.7,136.3,131.2,128.5,125.4,123.8,122.7,121.1$, 119.8, 117.9, 113.3, 108.2, 104.0, 97.0, 78.9, 51.1. HRMS (EI) Calcd for $\mathrm{C}_{18} \mathrm{H}_{13} \mathrm{NO}_{2}$ $\left(\mathrm{M}^{+}\right)$275.0946; Found, 275.0941 .
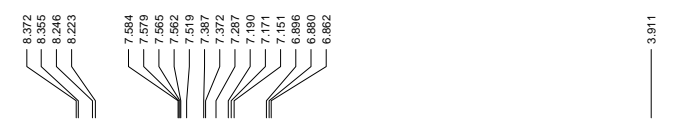

$3 b-\mathrm{H}$
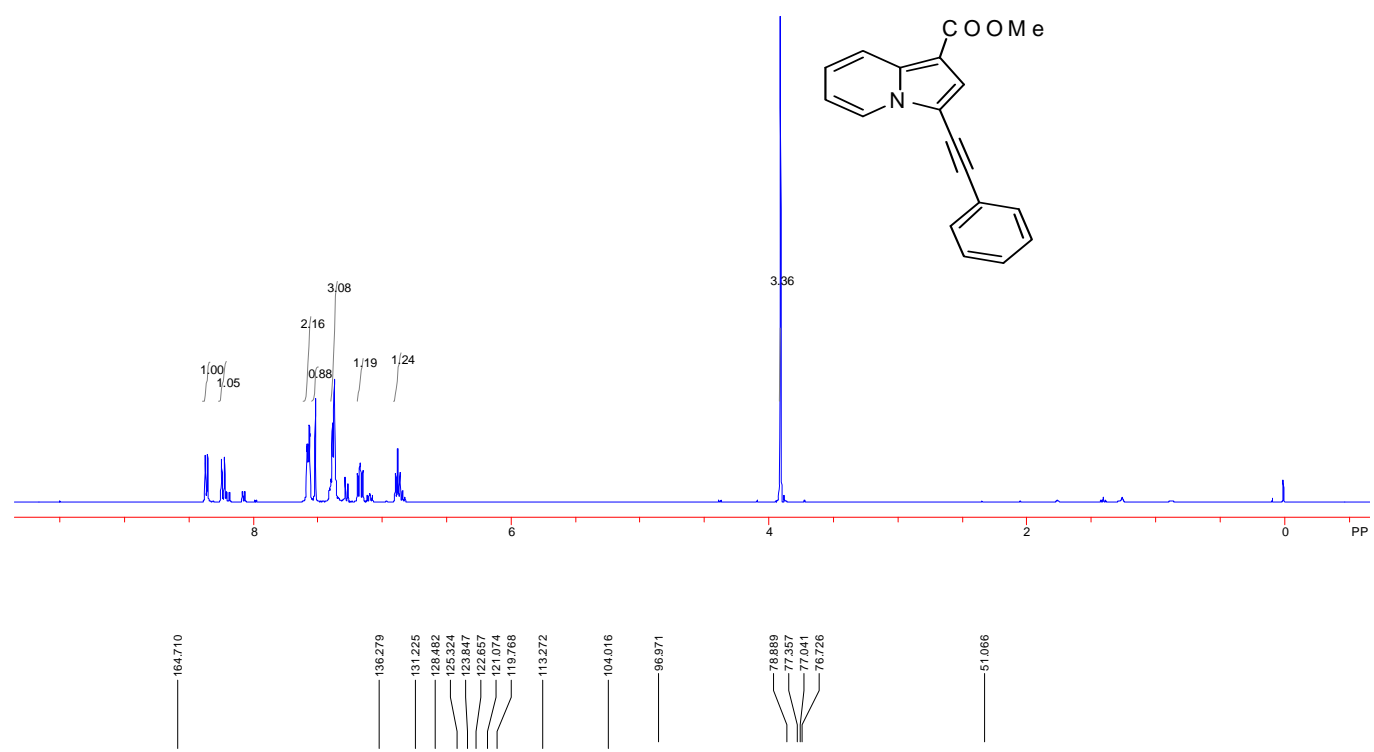

$3 b-C$

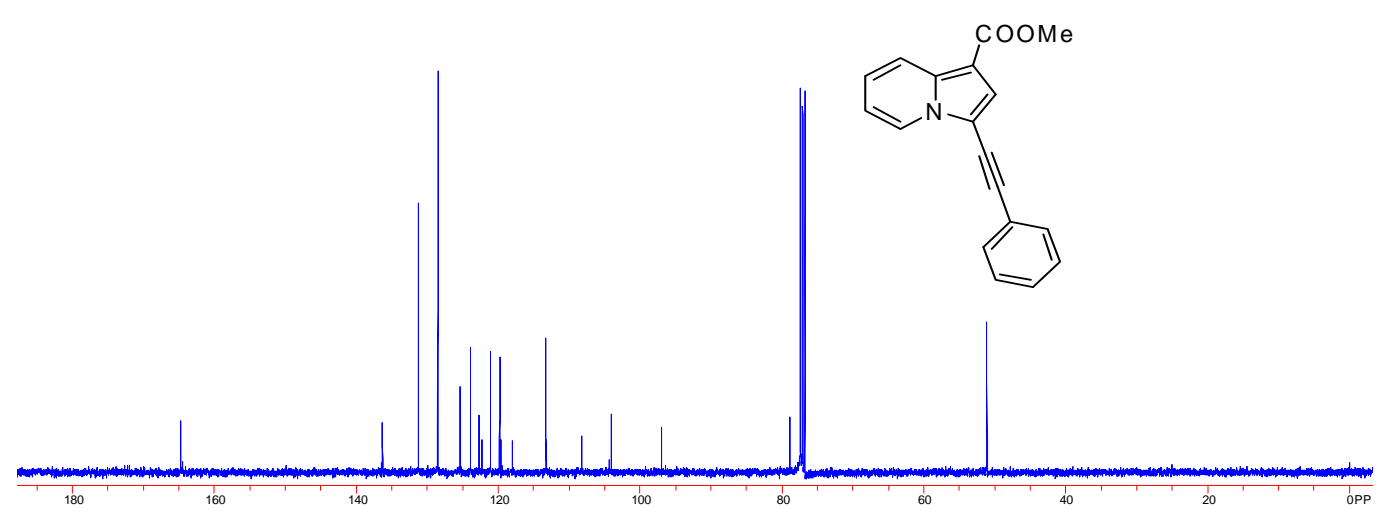




\section{Ethyl 3-(phenylethynyl)indolizine-1-carboxylate (3c ) ${ }^{[2]}$}

White solid; m.p. $247-248{ }^{\circ} \mathrm{C} ;{ }^{1} \mathrm{H}$ NMR ( $400 \mathrm{MHz}, \mathrm{CDCl}_{3}$, TMS ) $\delta 8.37$ ( d, $J=6.8$ $\mathrm{Hz}, 1 \mathrm{H}$ ), 8.24 ( d, $J=8.8 \mathrm{~Hz}, 1 \mathrm{H}$ ), 7.56-7.58 ( m, $2 \mathrm{H}$ ), 7.55 ( s, $1 \mathrm{H}$ ), 7.36-7.39 ( m, $3 \mathrm{H}), 7.17$ ( t $J=8.0 \mathrm{~Hz}, 1 \mathrm{H}), 6.88(\mathrm{t}, J=7.2 \mathrm{~Hz}, 1 \mathrm{H}), 4.36-4.41$ ( m, $2 \mathrm{H}$ ), $1.42(\mathrm{t}, J=7.2 \mathrm{~Hz}, 3 \mathrm{H}) ;{ }^{13} \mathrm{C} \mathrm{NMR}\left(100 \mathrm{MHz}, \mathrm{CDCl}_{3}\right) \delta 164.3,136.2,131.2,128.5$, 128.4, 125.3, 123.8, 122.7, 121.2, 119.8, 113.3, 108.1, 104.4, 97.0, 79.0, 59.7, 14.6. HRMS (EI) Calcd for $\mathrm{C}_{19} \mathrm{H}_{15} \mathrm{NO}_{2}\left(\mathrm{M}^{+}\right)$289.1103, Found 289.1106 .
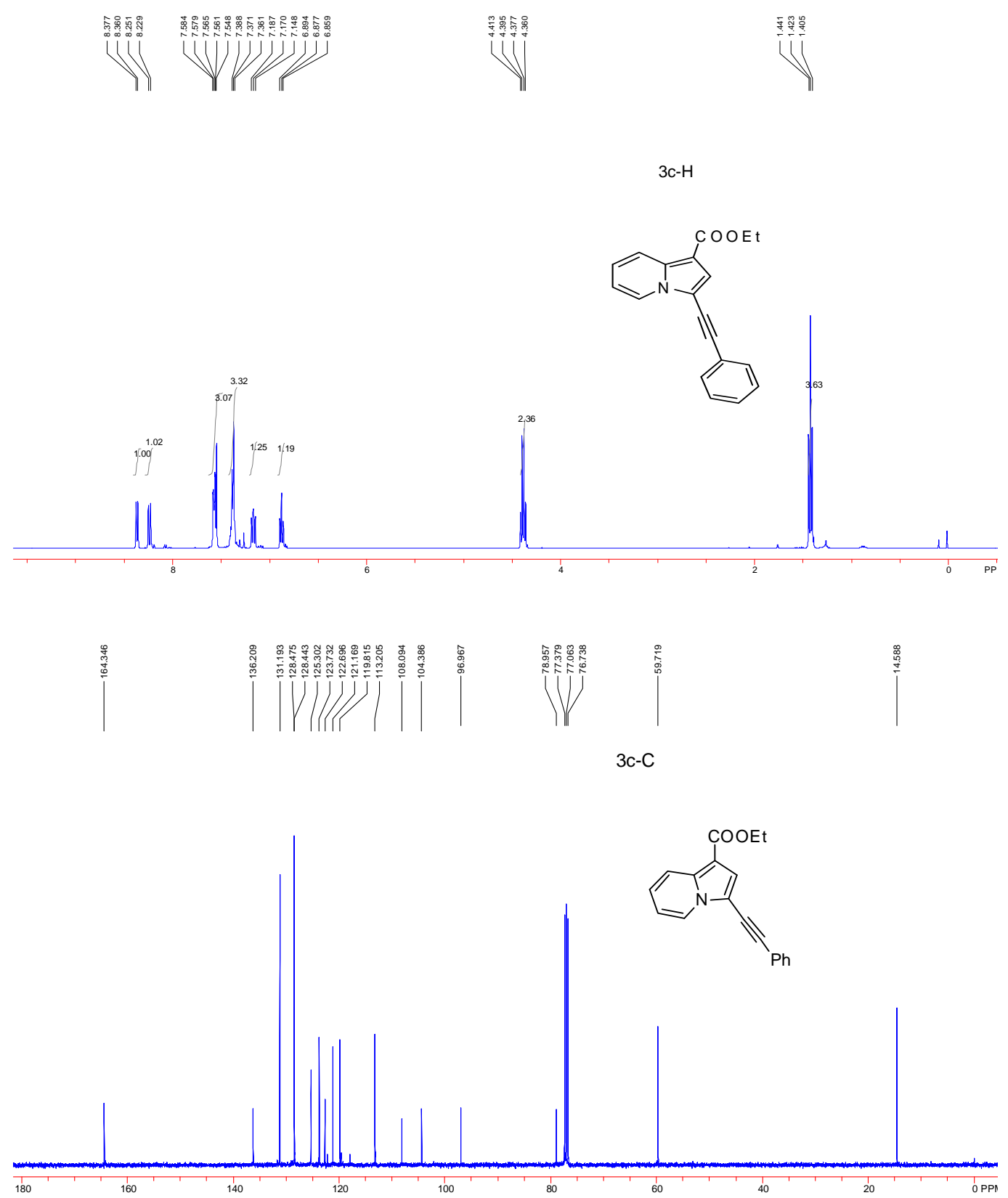


\section{n-Butyl 3-(phenylethynyl)indolizine-1-carboxylate (3d ) ${ }^{[2]}$}

Brown solid; m.p. 260-262 ${ }^{\circ} \mathrm{C}$; ${ }^{1} \mathrm{H}$ NMR ( $400 \mathrm{MHz}, \mathrm{CDCl}_{3}$, TMS ) $\delta 8.36$ ( d, $J=$ $6.8 \mathrm{~Hz}, 1 \mathrm{H}), 8.23(\mathrm{~d}, J=9.6 \mathrm{~Hz}, 1 \mathrm{H}), 7.55-7.58(\mathrm{~m}, 2 \mathrm{H}), 7.54(\mathrm{~s}, 1 \mathrm{H})$, 7.36-7.38 ( m, $3 \mathrm{H}$ ), 7.14-7.18 ( m, $1 \mathrm{H}$ ), 6.87 ( t, $J=7.6 \mathrm{~Hz}, 1 \mathrm{H}), 4.33$ ( t, $J=6.4$ $\mathrm{Hz}, 2 \mathrm{H}$ ), 1.74-1.79 ( m, $2 \mathrm{H}$ ), 1.48-1.54 ( m, $2 \mathrm{H}$ ), 1.00 ( t, J = 7.6 Hz, $3 \mathrm{H}$ ); 13C NMR ( $100 \mathrm{MHz}, \mathrm{CDCl} 3$ ) $\delta$ 164.4, 136.2, 131.2, 128.5, 128.4, 125.3, 123.7, 122.7, 121.2, 119.8, 113.2, 108.1, 104.4, 97.0, 78.9, 63.6, 31.0, 19.4, 13.8. HRMS (EI) Calcd for $\mathrm{C}_{21} \mathrm{H}_{19} \mathrm{NO}_{2}(\mathrm{M}+)$ 317.1416; Found, 317.1422.
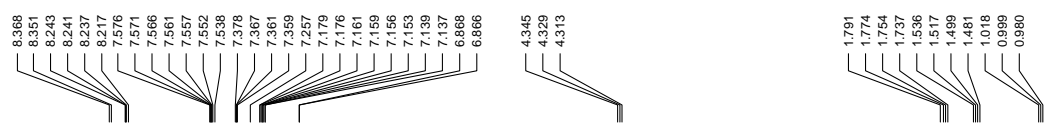

$3 d-\mathrm{H}$
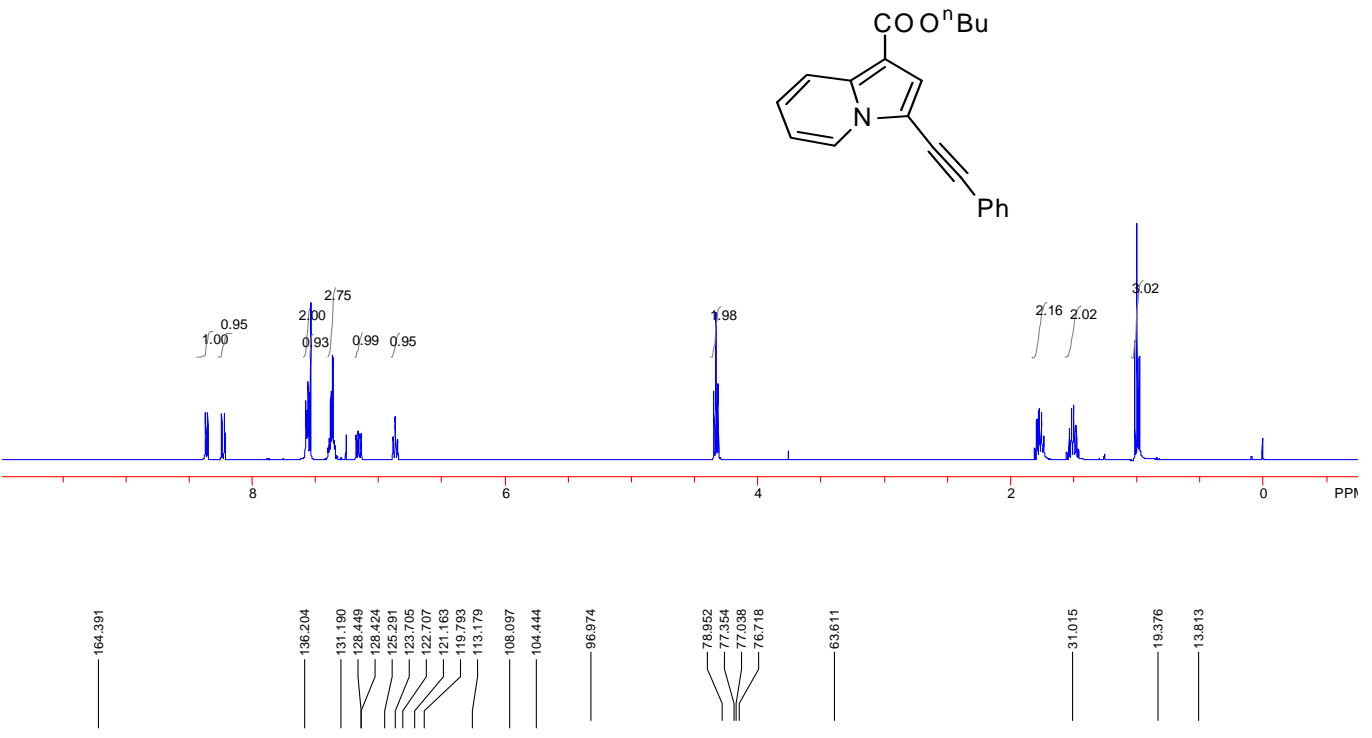

$3 d-C$

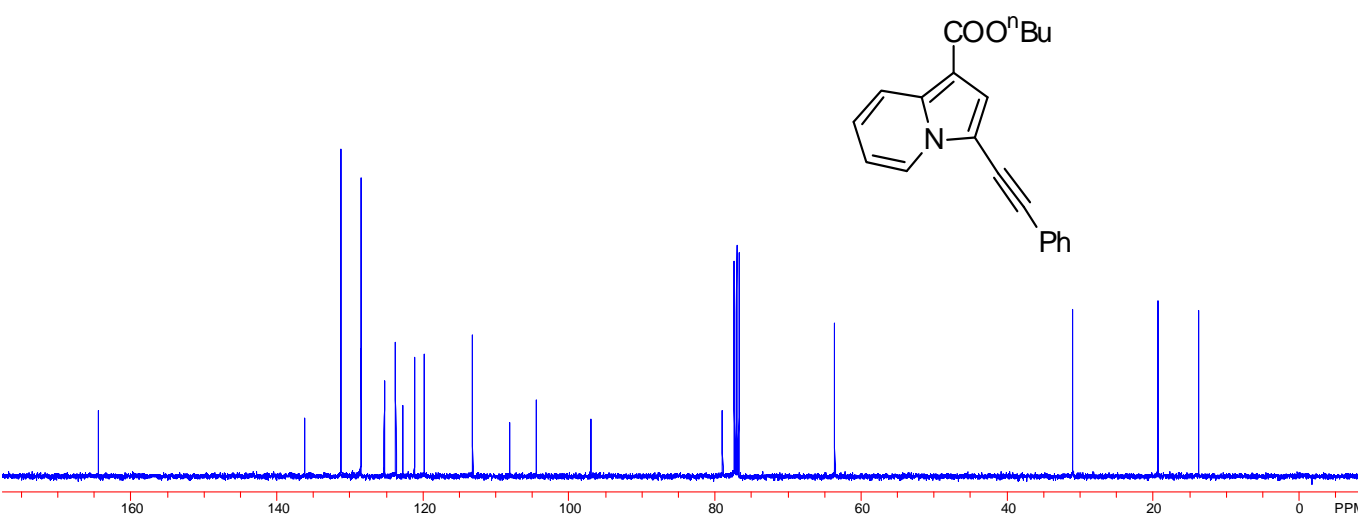




\section{2-Methyl-3-(phenylethynyl)indolizine-1-carbonitrile ( 3e ) ${ }^{[2]}$}

White solid; m.p. $293-294{ }^{\circ} \mathrm{C}$; ${ }^{1} \mathrm{H}$ NMR ( $400 \mathrm{MHz}, \mathrm{CDCl}_{3}$, TMS ) $\delta 8.27$ ( d, J=7.2 $\mathrm{Hz}, 1 \mathrm{H}$ ), 7.54-7.58 ( m, $3 \mathrm{H}$ ), 7.39-7.40 ( m, $3 \mathrm{H}$ ), 7.13 ( t, J=7.6 Hz, $1 \mathrm{H}$ ), 6.86 $(\mathrm{t}, J=7.6 \mathrm{~Hz}, 1 \mathrm{H}), 2.52(\mathrm{~s}, 3 \mathrm{H}) ;{ }^{13} \mathrm{C} \mathrm{NMR}\left(100 \mathrm{MHz}, \mathrm{CDCl}_{3}\right) \delta 137.4,133.5$, $131.2,128.7,128.5,125.5,123.8,122.4,117.1,116.0,113.3,107.7,99.6,83.4,77.3$, 11.4. HRMS (EI) Calcd for $\mathrm{C}_{18} \mathrm{H}_{12} \mathrm{~N}_{2}\left(\mathrm{M}^{+}\right)$256.1000; Found, 256.1001.
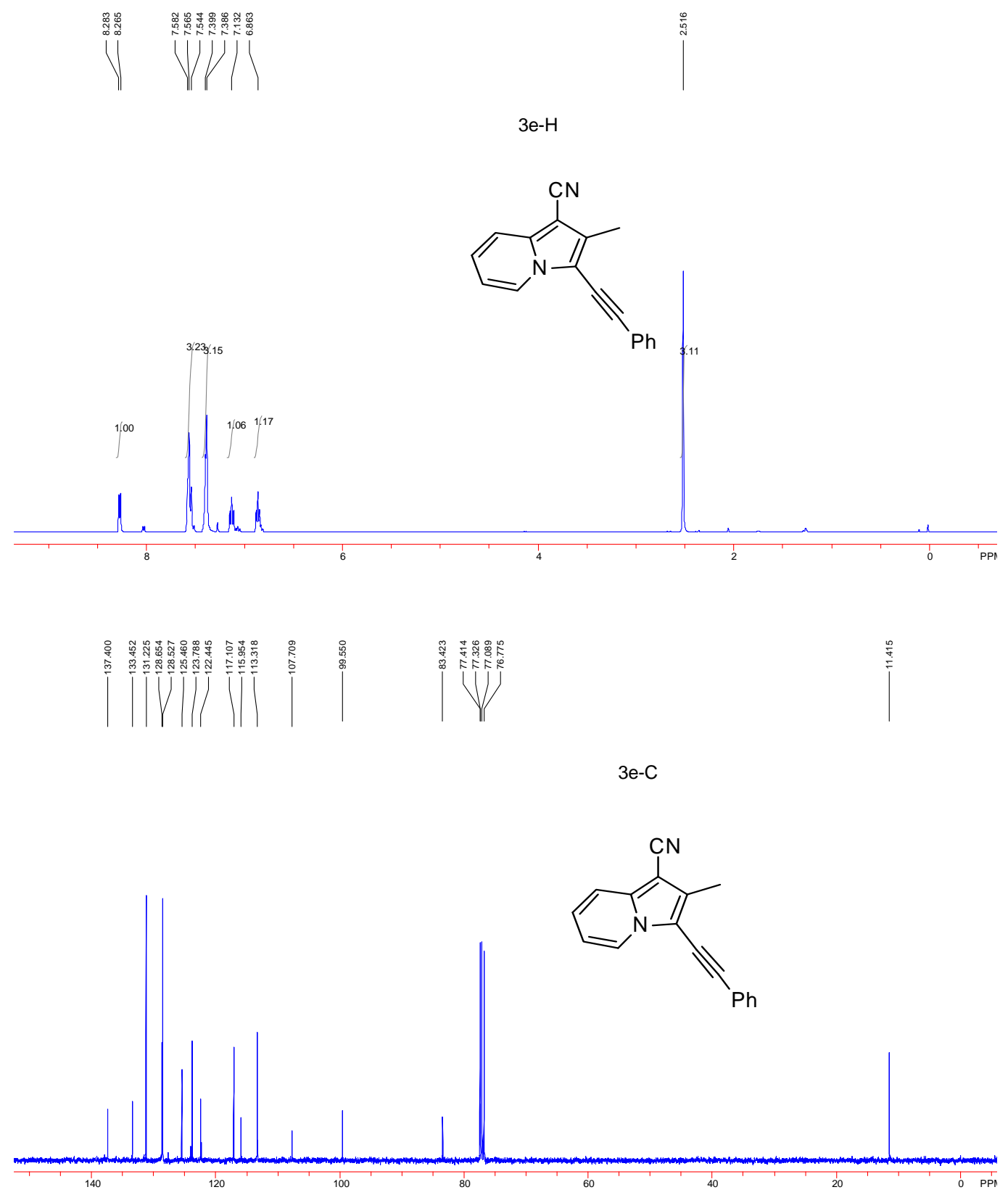
3-(o-tolylethynyl)indolizine-1-carbonitrile ( 4a )

Brown solid; mp 158.9-160.2 ${ }^{\circ} \mathrm{C} ;{ }^{1} \mathrm{H}$ NMR ( $400 \mathrm{MHz}, \mathrm{CDCl}_{3}$, TMS ) $\delta 8.38$ ( d, $J=8$ $\mathrm{Hz}, 1 \mathrm{H}$ ), 7.70 ( d, $J=8 \mathrm{~Hz}, 1 \mathrm{H}$ ), 7.54 (d, $J=8 \mathrm{~Hz}, 1 \mathrm{H}), 7.28-7.30$ ( m, $3 \mathrm{H}$ ), 7.20-7.22 ( m, $2 \mathrm{H}$ ), $6.95(\mathrm{t}, J=6.4 \mathrm{~Hz}, 1 \mathrm{H}$ ), 2.56(s, $3 \mathrm{H}) ;{ }^{13} \mathrm{C}$ NMR ( $100 \mathrm{MHz}$, $\left.\mathrm{CDCl}_{3}\right) \delta 138.7,137.0,130.8,128.7,128.0,124.8,124.6,122.8,121.0,120.1,116.9$, 115.1, 112.8, 95.2, 81.5, 80.5, 20.0; IR (neat) v 3105, 2215, 1596, 1504, 1458, 785, $747 \mathrm{~cm}^{-1}$; HRMS (EI) Calcd for $\mathrm{C}_{18} \mathrm{H}_{12} \mathrm{~N}_{2}\left(\mathrm{M}^{+}\right)$256.1000; Found, 256.1002.
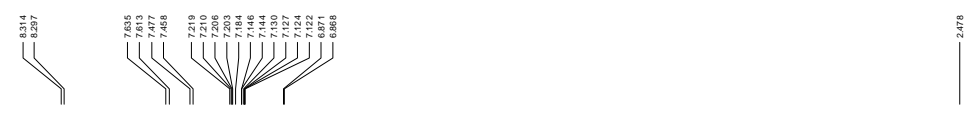

SZQ-481-67-H
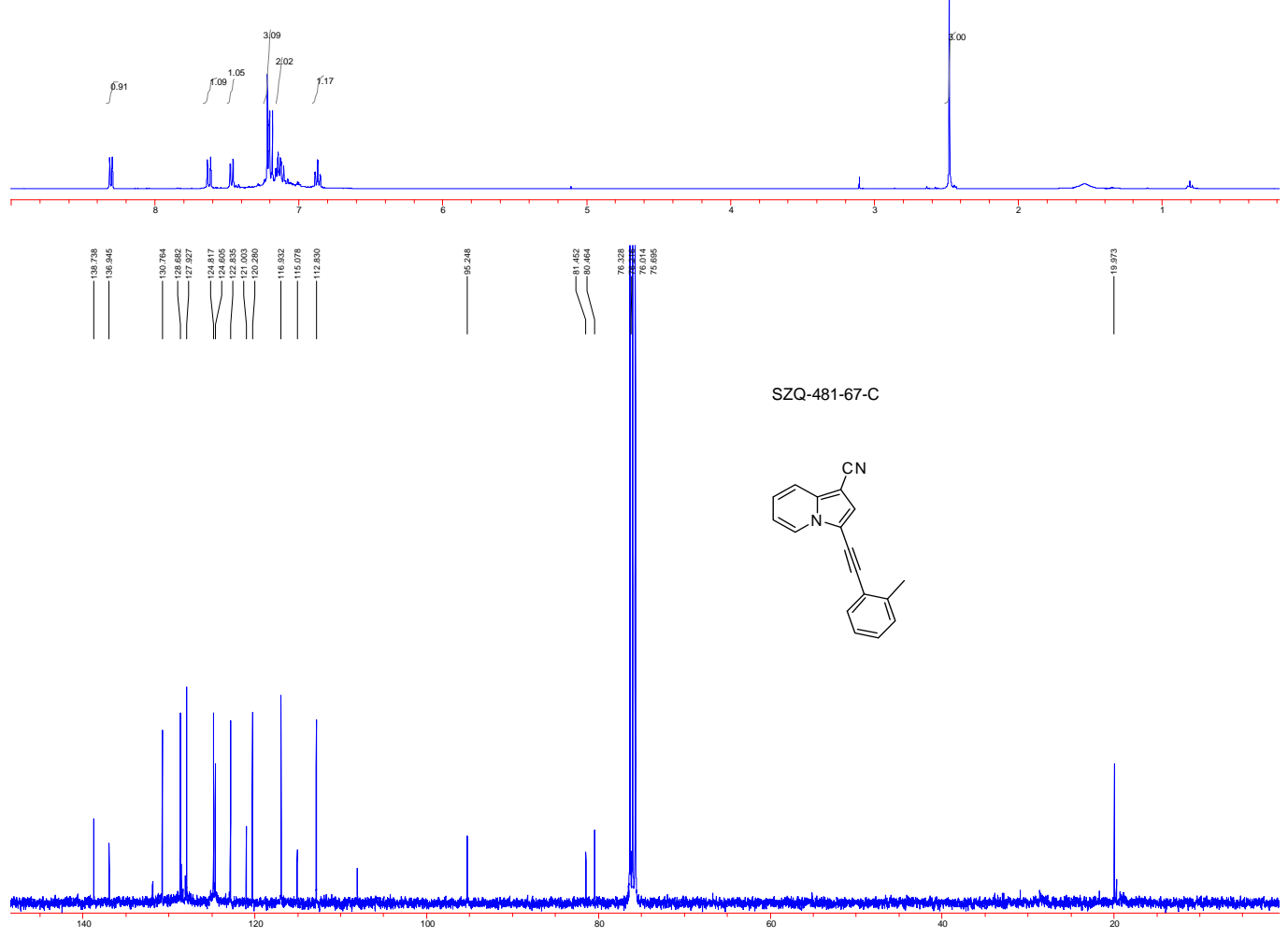

9 


\section{3-(m-tolylethynyl)indolizine-1-carbonitrile ( 4b )}

Brown solid; mp 160.5-161.5 ${ }^{\circ}$; ${ }^{1} \mathrm{H}$ NMR ( $400 \mathrm{MHz}, \mathrm{CDCl}_{3}$, TMS ) $\delta 8.34$ ( d, $J=$ $6.8 \mathrm{~Hz}, 1 \mathrm{H}$ ), 7.64 ( d, $J=8.8 \mathrm{~Hz}, 1 \mathrm{H}$ ), 7.33-7.36 ( m, $2 \mathrm{H}$ ), 7.22-7.24 ( m, $2 \mathrm{H}$ ), 7.13-7.17 ( m, $2 \mathrm{H}$ ), 6.89 ( t, $J=6.4 \mathrm{~Hz}, 1 \mathrm{H}$ ), 2.31(s, $3 \mathrm{H}) ;{ }^{13} \mathrm{C}$ NMR ( $100 \mathrm{MHz}$, $\left.\mathrm{CDCl}_{3}\right) \delta 138.3,131.9,129.8,128.5,125.8,123.9,122.5,122.0,121.3,117.9,113.8$, 97.6, 82.4, 21.3; IR (neat) v 3100, 2215, 1602, 1508, 1463, 785, $747 \mathrm{~cm}^{-1}$; HRMS (EI) Calcd for $\mathrm{C}_{18} \mathrm{H}_{12} \mathrm{~N}_{2}\left(\mathrm{M}^{+}\right)$256.1000; Found, 256.1002.

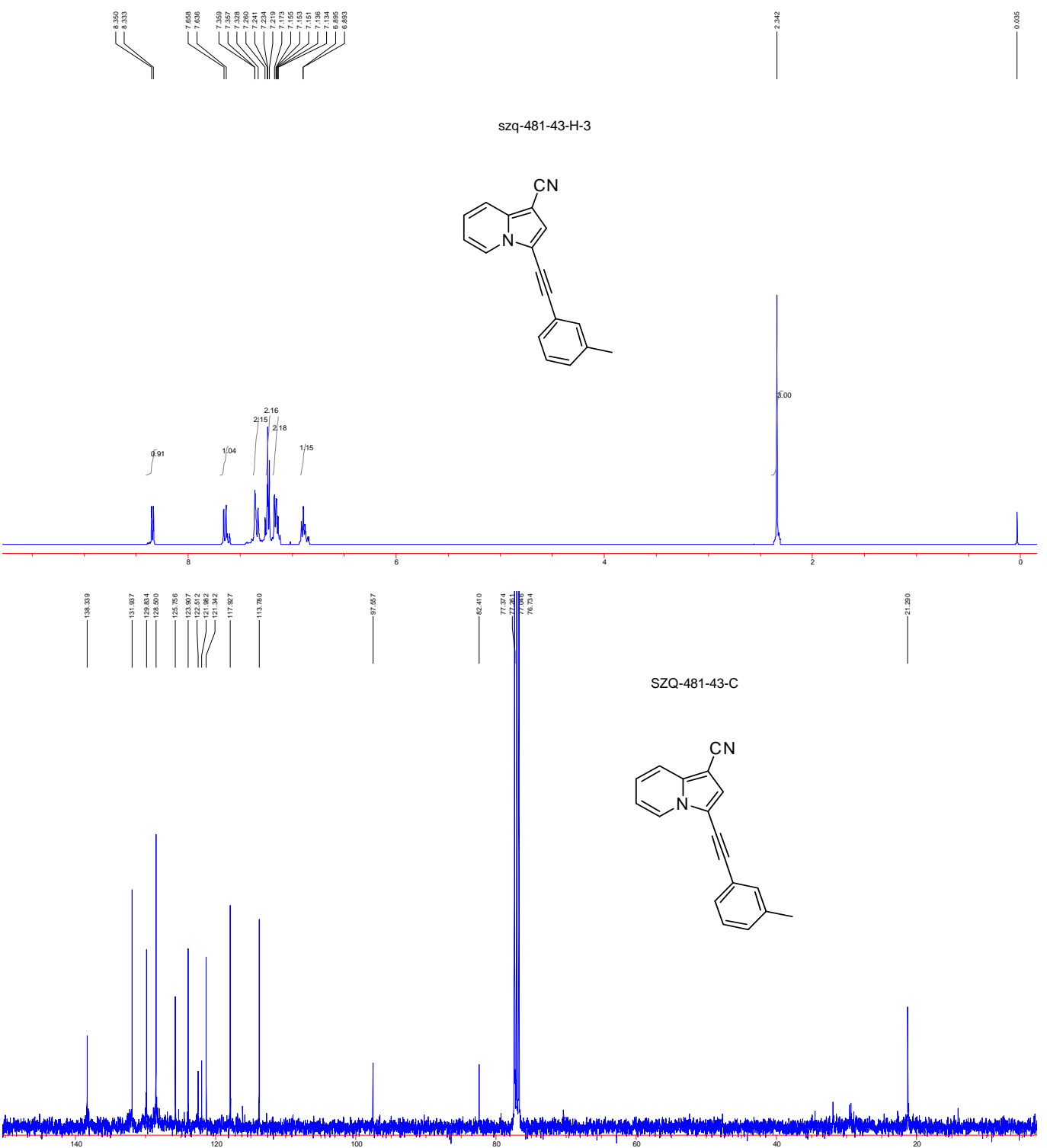




\section{3-((3-fluorophenyl)ethynyl)indolizine-1-carbonitrile ( 4c )}

Brown solid; mp 161.2-162. ${ }^{\circ} \mathrm{C}$; ${ }^{1} \mathrm{H}$ NMR ( $400 \mathrm{MHz}, \mathrm{CDCl}_{3}$, TMS ) $\delta 8.37$ ( d, $J=8$ $\mathrm{Hz}, 1 \mathrm{H}), 7.71(\mathrm{~d}, J=8 \mathrm{~Hz}, 1 \mathrm{H}), 7.35-7.38(\mathrm{~m}, 2 \mathrm{H}), 7.31(\mathrm{~s}, 1 \mathrm{H}), 7.20-7.28(\mathrm{~m}$, $2 \mathrm{H}), 7.07-7.12(\mathrm{t}, J=8 \mathrm{~Hz}, 1 \mathrm{H}), 6.96(\mathrm{t}, J=8 \mathrm{~Hz}, 1 \mathrm{H}) ;{ }^{13} \mathrm{C}$ NMR $(100 \mathrm{MHz}$, $\left.\mathrm{CDCl}_{3}\right) \delta 162.4(\mathrm{~d}, J=245.3 \mathrm{~Hz}), 138.1,130.2(\mathrm{~d}, J=9.5 \mathrm{~Hz}), 127.2(\mathrm{~d}, J=3.4 \mathrm{~Hz})$, 125.7, 124.2, 121.9, 118.1(d, $J=22.1 \mathrm{~Hz}), 118.0,116.2(\mathrm{~d}, J=22 \mathrm{~Hz}), 115.9,114.0$, 108.4, 96.2, 82.7, 78.7; IR (neat) v 3104, 2214, 1608, 1579, 1508, $1246 \mathrm{~cm}^{-1}$; HRMS (EI) Calcd for $\mathrm{C}_{17} \mathrm{H}_{9} \mathrm{FN}_{2}\left(\mathrm{M}^{+}\right)$260.0750; Found, 260.0750.
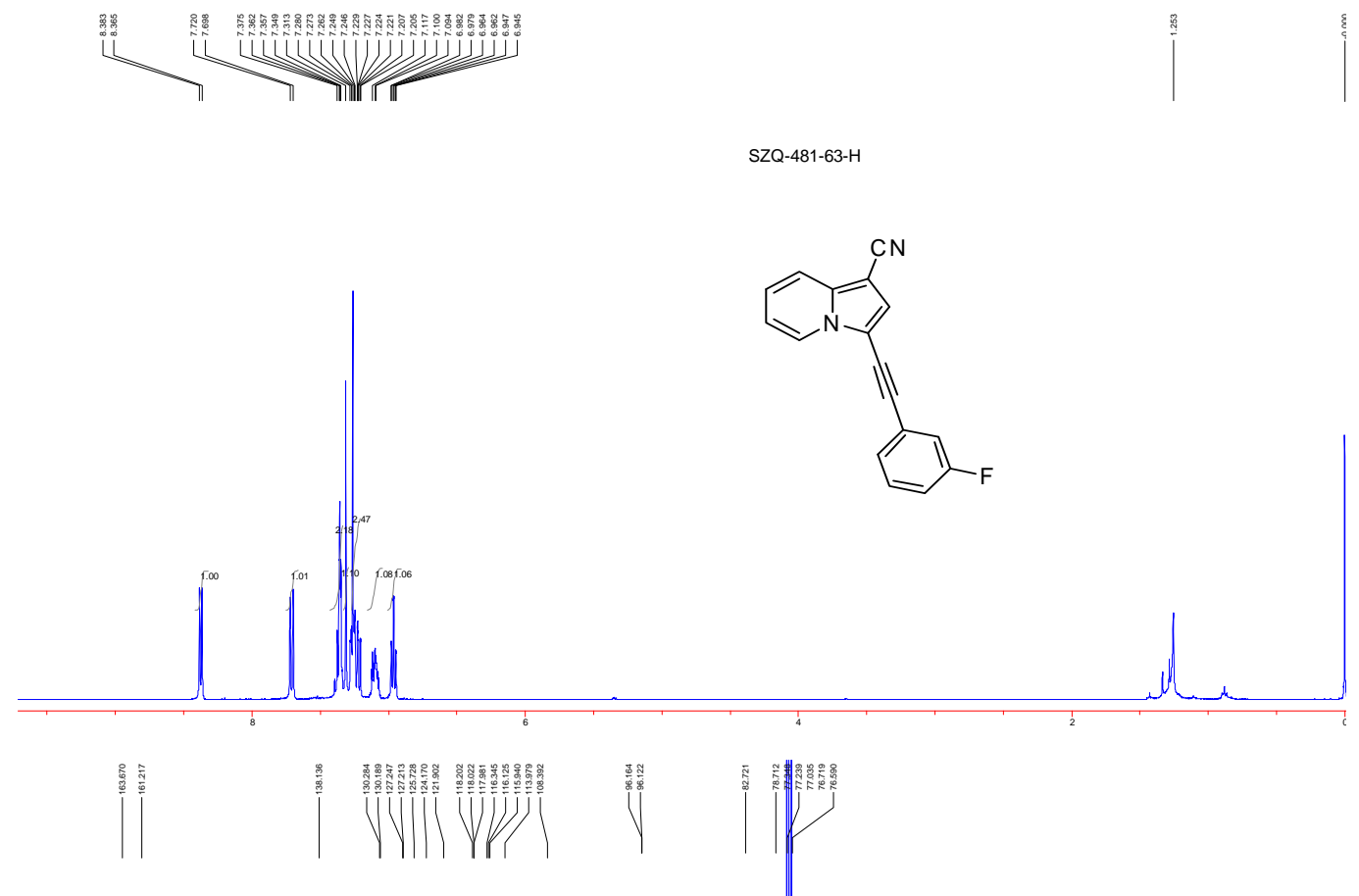

SZQ-481-63-C-2

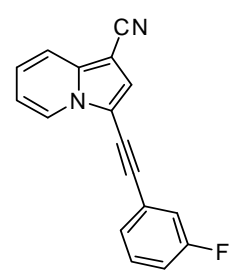




\section{3-((2-fluorophenyl)ethynyl)indolizine-1-carbonitrile (4d )}

Brown solid; mp 160.3-161.4 ${ }^{\circ} \mathrm{C} ;{ }^{1} \mathrm{H}$ NMR ( $400 \mathrm{MHz}, \mathrm{CDCl}_{3}$, TMS ) $\delta 8.36$ ( d, $J=8$ $\mathrm{Hz}, 1 \mathrm{H}$ ), 7.68 ( d, $J=8 \mathrm{~Hz}, 1 \mathrm{H}$ ), 7.34-7.36 ( m, $2 \mathrm{H}$ ), 7.30 ( s, $1 \mathrm{H}$ ), 7.22-7.26 ( m, $2 \mathrm{H}), 7.10(\mathrm{~m}, 1 \mathrm{H}), 6.96(\mathrm{t}, J=8 \mathrm{~Hz}, 1 \mathrm{H}) ;{ }^{13} \mathrm{C} \mathrm{NMR}\left(100 \mathrm{MHz}, \mathrm{CDCl}_{3}\right) \delta$ $162.1(\mathrm{~d}, J=243.5 \mathrm{~Hz}), 138.0,129.8(\mathrm{~d}, J=10.1 \mathrm{~Hz}), 127.20(\mathrm{~d}, J=3.6 \mathrm{~Hz}), 125.4$, 124.1, 121.6, 117.9(d, $J=22.4 \mathrm{~Hz}), 118.0,116.4(\mathrm{~d}, J=22.6 \mathrm{~Hz}), 115.7,113.8,108.4$, 96.2, 82.5, 78.6; IR (neat) v 3102, 2231, 1612, 1574, 1502, $1238 \mathrm{~cm}^{-1}$; HRMS (EI) Calcd for $\mathrm{C}_{17} \mathrm{H}_{9} \mathrm{FN}_{2}\left(\mathrm{M}^{+}\right)$260.0750; Found, 260.0750.

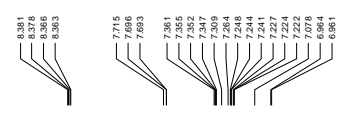

SZQ-481-47-H
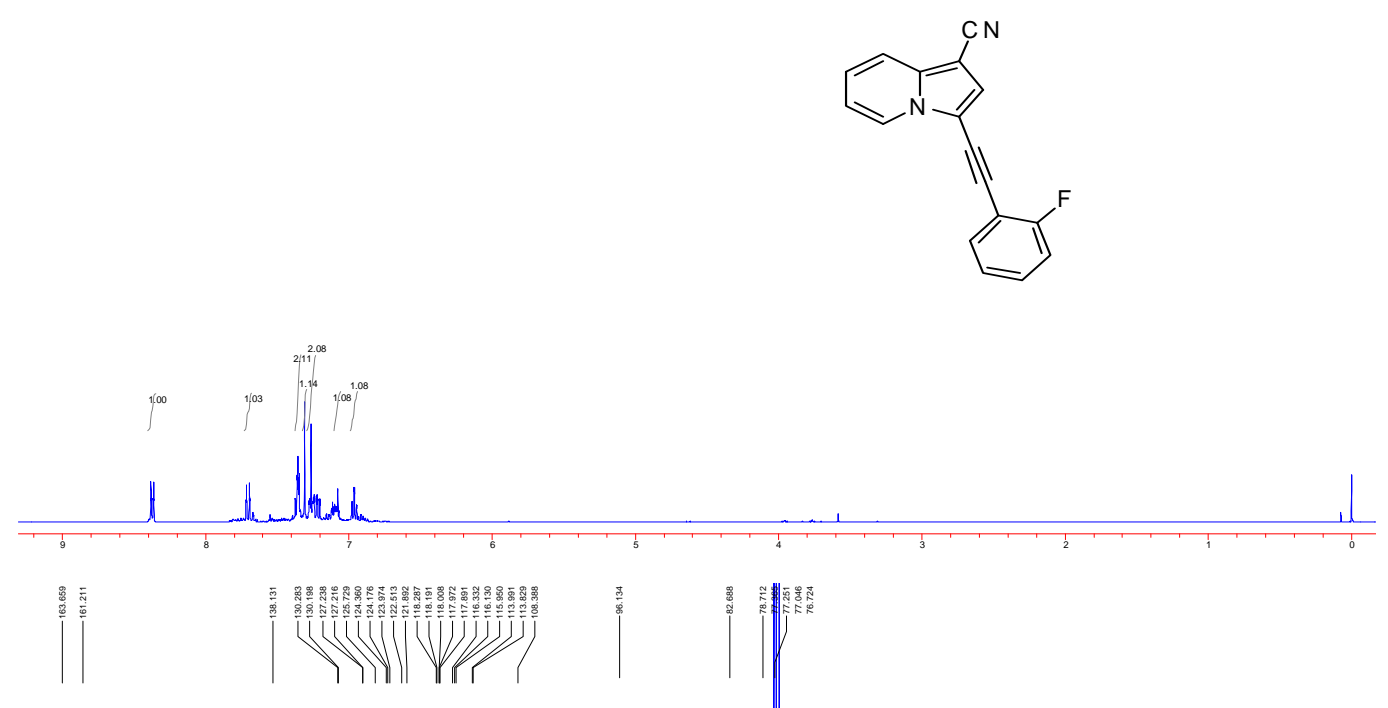

szq-481-47-C

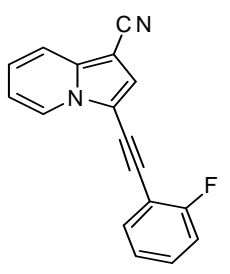




\section{3-((4-chlorophenyl)ethynyl)indolizine-1-carbonitrile ( 4e ) ${ }^{[2]}$}

Yellow solid; m.p. $317-318{ }^{\circ} \mathrm{C} ;{ }^{1} \mathrm{H}$ NMR ( $400 \mathrm{MHz}, \mathrm{CDCl}_{3}$, TMS ) $\delta 8.36$ ( d, $J=$ $7.2 \mathrm{~Hz}, 1 \mathrm{H}$ ), 7.69( d, $J=8.8 \mathrm{~Hz}, 1 \mathrm{H}$ ), 7.49 ( d, $J=8.0 \mathrm{~Hz}, 2 \mathrm{H}$ ), $7.36(\mathrm{~d}, J=8.4$ $\mathrm{Hz}, 2 \mathrm{H}$ ), 7.28 ( s, $1 \mathrm{H}), 7.21(\mathrm{t}, J=8.0 \mathrm{~Hz}, 1 \mathrm{H}), 6.95(\mathrm{t}, J=6.8 \mathrm{~Hz}, 1 \mathrm{H}) ;{ }^{13} \mathrm{C}$ NMR ( $\left.100 \mathrm{MHz}, \mathrm{CDCl}_{3}\right) \delta 138.0,134.9,132.5,128.9,125.7,124.1,121.7,120.6$, 117.9, 115.9, 113.9, 108.5, 96.2, 82.5, 78.7. HRMS (EI) Calcd for $\mathrm{C}_{16} \mathrm{H}_{12} \mathrm{~N}_{2}\left(\mathrm{M}^{+}\right)$ 276.0454, Found 276.0457.
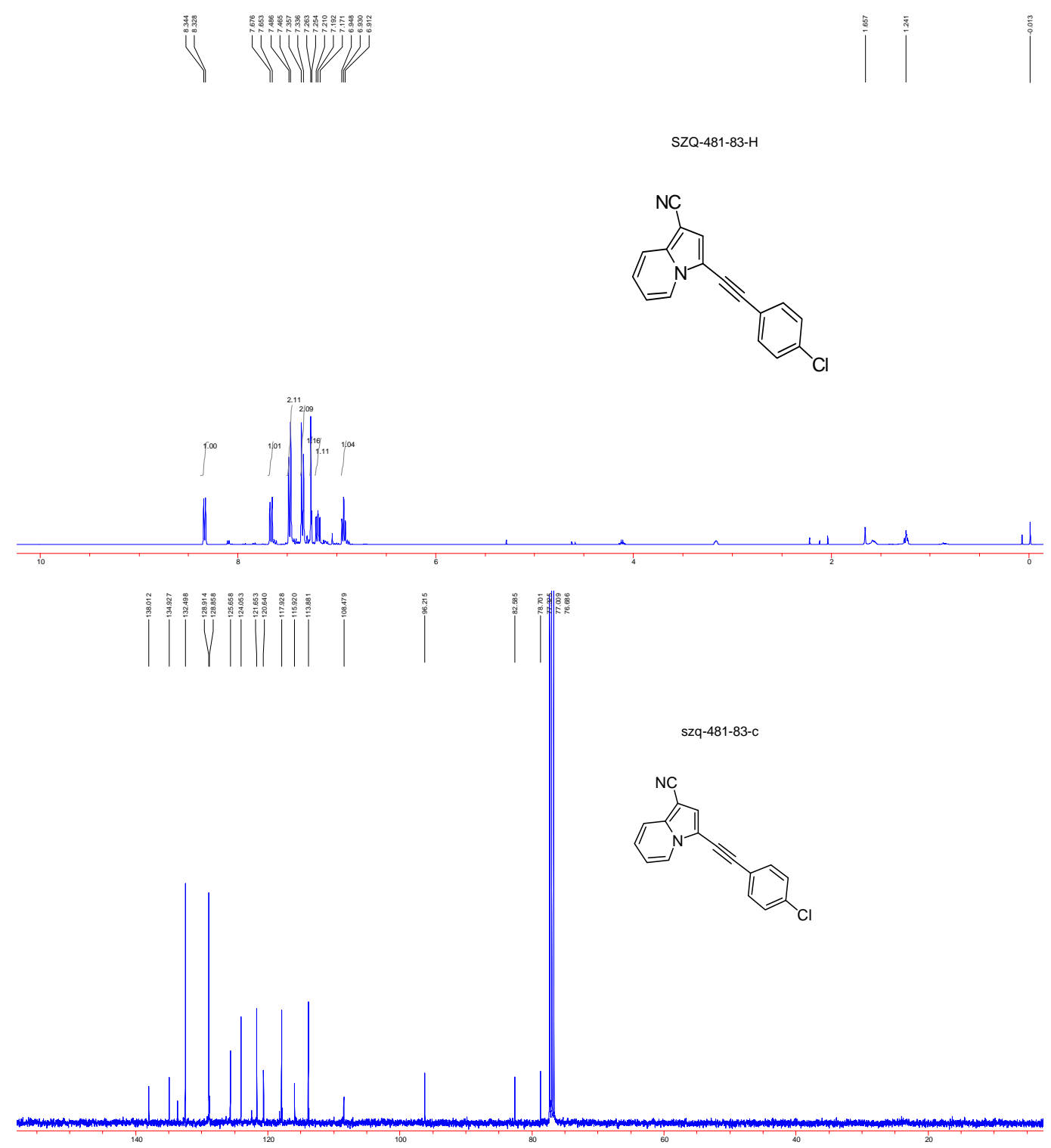


\section{3-((4-methoxyphenyl)ethynyl)indolizine-1-carbonitrile ( $4 \mathrm{f})^{\text {[2] }}$}

White solid; m.p. $296-297{ }^{\circ} \mathrm{C} ;{ }^{1} \mathrm{H}$ NMR ( $400 \mathrm{MHz}, \mathrm{CDCl}_{3}$, TMS ) $\delta 8.37$ ( d, $J=8$ $\mathrm{Hz}, 1 \mathrm{H}), 7.68(\mathrm{~d}, J=8 \mathrm{~Hz}, 1 \mathrm{H}), 7.50-7.53$ ( m, $2 \mathrm{H}$ ), 7.27 ( s, $1 \mathrm{H}), 7.19$ (t, $J=8$ $\mathrm{Hz}, 1 \mathrm{H}$ ), 6.90-6.93 (m, $3 \mathrm{H}), 3.86(\mathrm{~s}, 3 \mathrm{H}) ;{ }^{13} \mathrm{C} \mathrm{NMR}\left(100 \mathrm{MHz}, \mathrm{CDCl}_{3}\right) \delta 160.2$, 137.9, 133.1, 125.7, 123.7, 121.0, 117.9, 114.2, 114.1, 113.6, 109.2, 97.2, 82.3, 55.4. HRMS (EI) Calcd for $\mathrm{C}_{18} \mathrm{H}_{12} \mathrm{~N}_{2} \mathrm{O}\left(\mathrm{M}^{+}\right)$272.0950; Found, 272.0950.

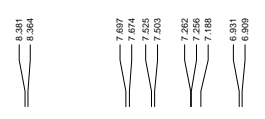

$$
\text { szq-481-74-H }
$$
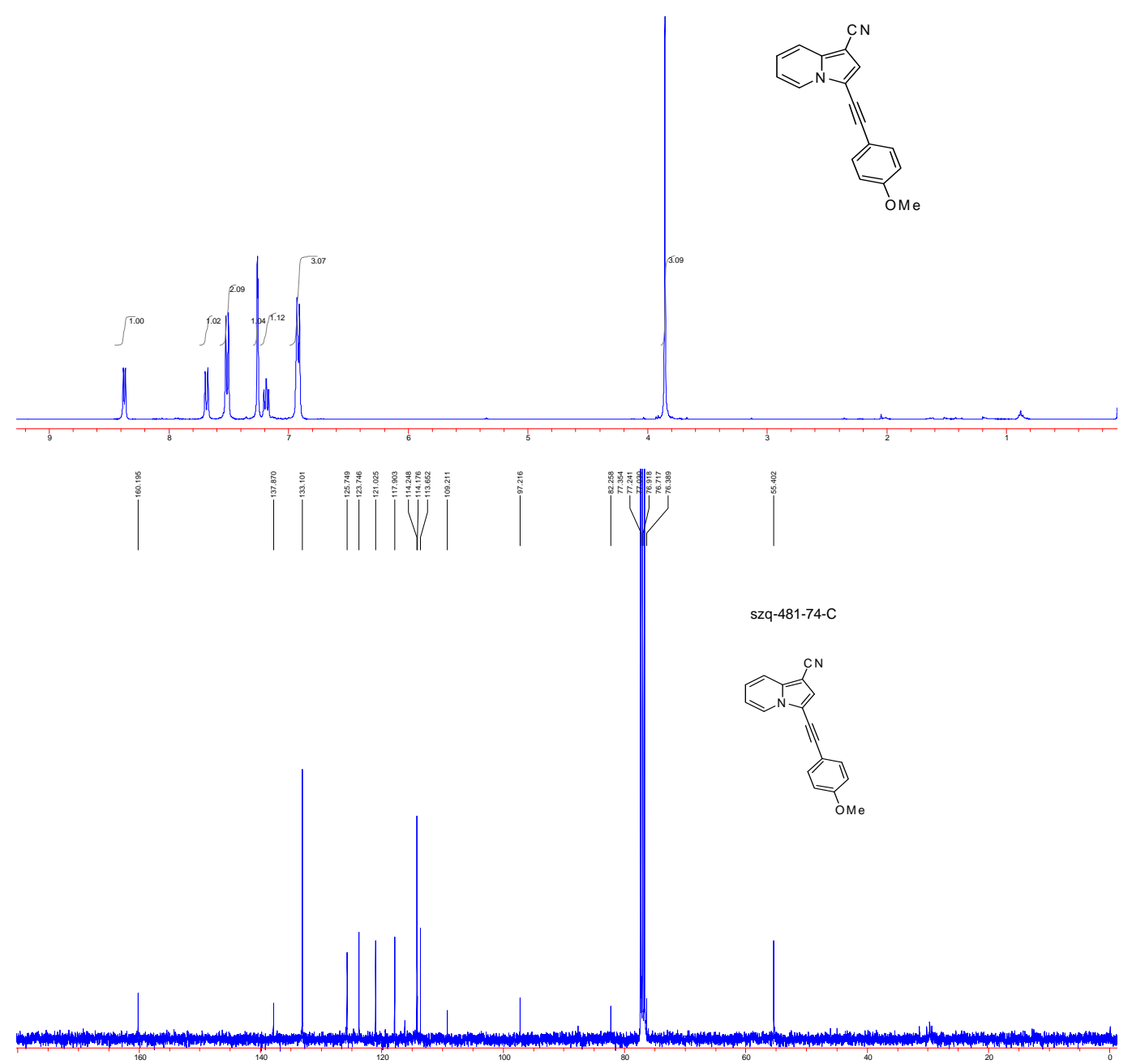


\section{3-((4-(tert-butyl)phenyl)ethynyl)indolizine-1-carbonitrile ( $4 \mathrm{~g}$ )}

Brown solid; mp 164.0-165.1 ${ }^{\circ} \mathrm{C} ;{ }^{1} \mathrm{H}$ NMR ( $400 \mathrm{MHz}, \mathrm{CDCl}_{3}$, TMS ) $\delta 8.37$ ( d, $J=8$ $\mathrm{Hz}, 1 \mathrm{H}), 7.68$ ( d, $J=8 \mathrm{~Hz}, 1 \mathrm{H}$ ), 7.50-7.52 ( m, $2 \mathrm{H}$ ), 7.40-7.43 ( m, $2 \mathrm{H}$ ), 7.27 ( s, $1 \mathrm{H}), 7.19(\mathrm{t}, J=8 \mathrm{~Hz}, 1 \mathrm{H}), 6.93(\mathrm{t}, J=8 \mathrm{~Hz}, 1 \mathrm{H}) ;{ }^{13} \mathrm{C} \mathrm{NMR}\left(100 \mathrm{MHz}, \mathrm{CDCl}_{3}\right)$ $\delta 152.4,137.9,131.2,125.8,125.6,123.9,121.2,119.1,117.9,116.2,113.7,109.1$, 97.5, 82.3, 34.9, 31.2; IR (neat) $v$ 3098, 2923, 2216, 1511, 1463, 835, $747 \mathrm{~cm}^{-1}$; HRMS (EI) Calcd for $\mathrm{C}_{21} \mathrm{H}_{18} \mathrm{~N}_{2}\left(\mathrm{M}^{+}\right)$298.1470; Found, 298.1465.

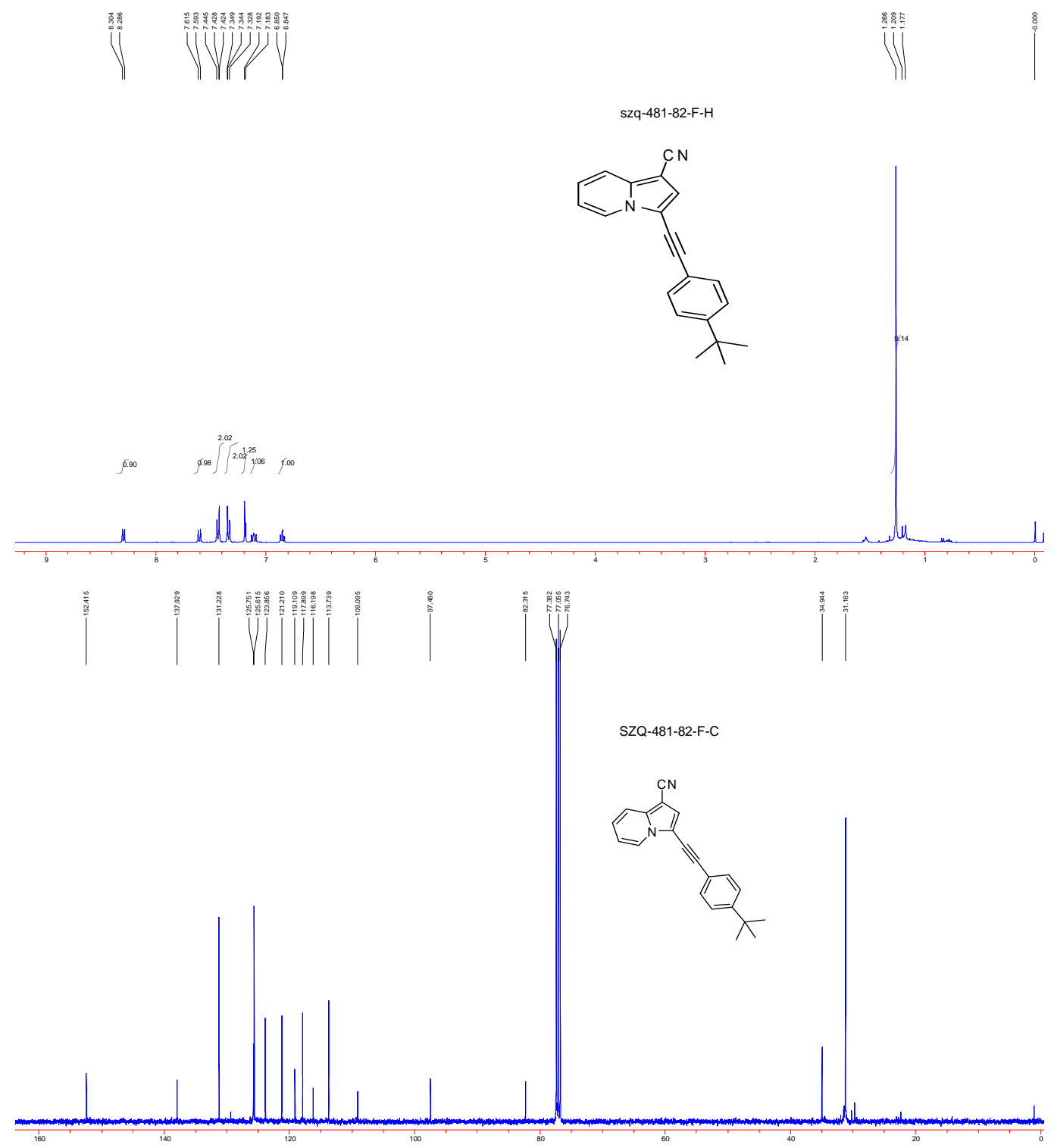




\section{3-((3-nitrophenyl)ethynyl)indolizine-1-carbonitrile (4h)}

Brown solid; mp 175.4-176.3 ${ }^{\circ} \mathrm{C} ;{ }^{1} \mathrm{H}$ NMR ( $400 \mathrm{MHz}, \mathrm{CDCl}_{3}$, TMS ) $\delta 8.40$ ( m, 2 $\mathrm{H}), 8.23(\mathrm{~d}, J=8 \mathrm{~Hz}, 1 \mathrm{H}), 7.86(\mathrm{~d}, J=8 \mathrm{~Hz}, 1 \mathrm{H}), 7.73(\mathrm{~d}, 1 \mathrm{H}), 7.60$ ( t, $J=8$ $\mathrm{Hz}, 1 \mathrm{H}), 7.37(\mathrm{~s}, 1 \mathrm{H}), 7.25(\mathrm{~m}, 1 \mathrm{H}), 7.01(\mathrm{t}, J=8 \mathrm{~Hz}, 1 \mathrm{H}) ;{ }^{13} \mathrm{C}$ NMR $(100$ $\left.\mathrm{MHz}, \mathrm{CDCl}_{3}\right) \delta 138.4,136.7,129.7,126.0,125.8,124.5,124.1,123.9,123.3,122.6$, 118.1, 115.7, 114.3, 107.8, 95.1, 83.1, 80.5; IR (neat) v 3100, 2214, 1602, 1521, 1463, 1345, 903, $745 \mathrm{~cm}-1$; HRMS (EI) Calcd for $\mathrm{C}_{17} \mathrm{H}_{9} \mathrm{~N}_{3} \mathrm{O}_{2}\left(\mathrm{M}^{+}\right)$287.0695; Found, 287.0697 .
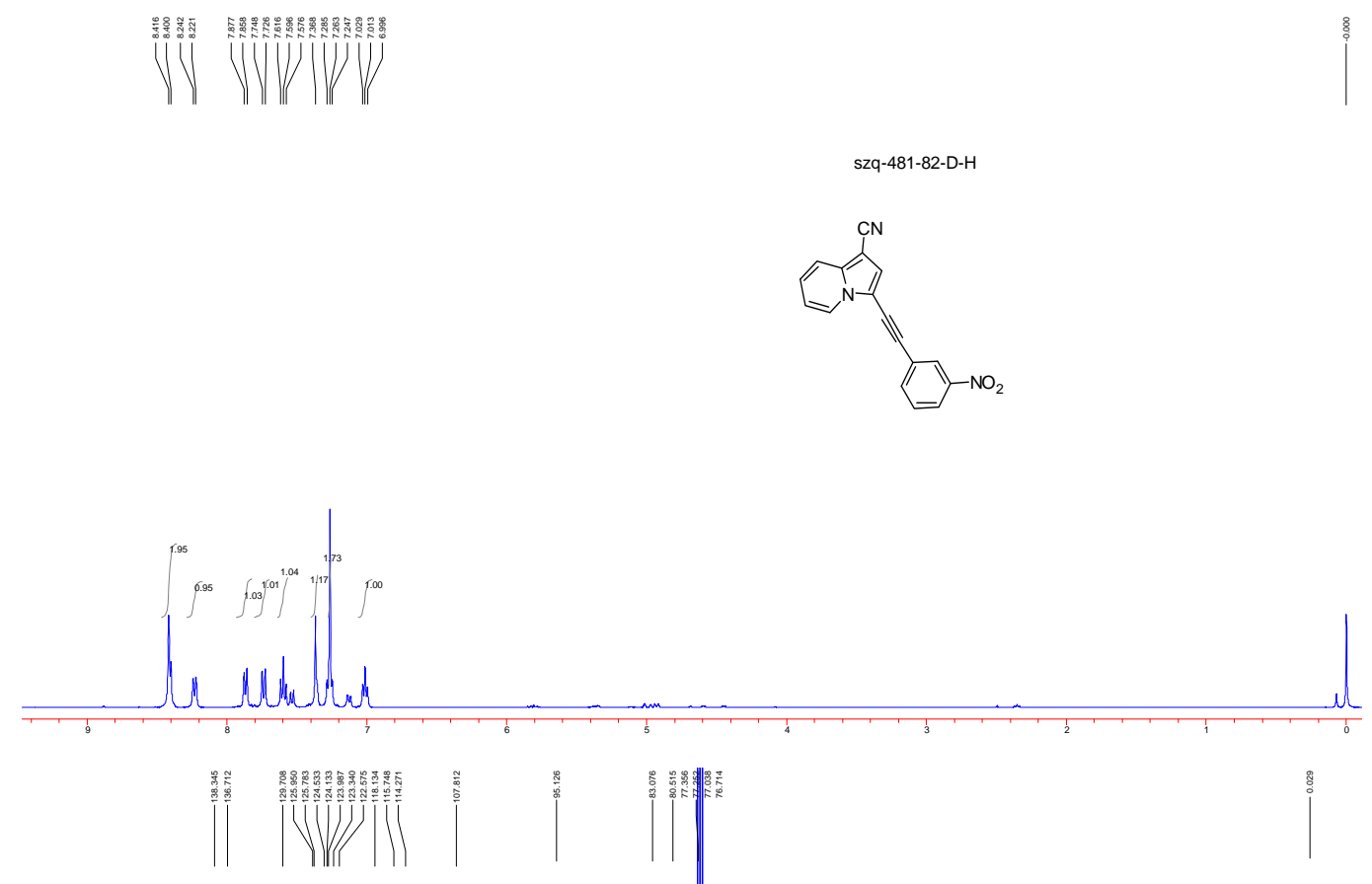

SZQ-481-82-D-C
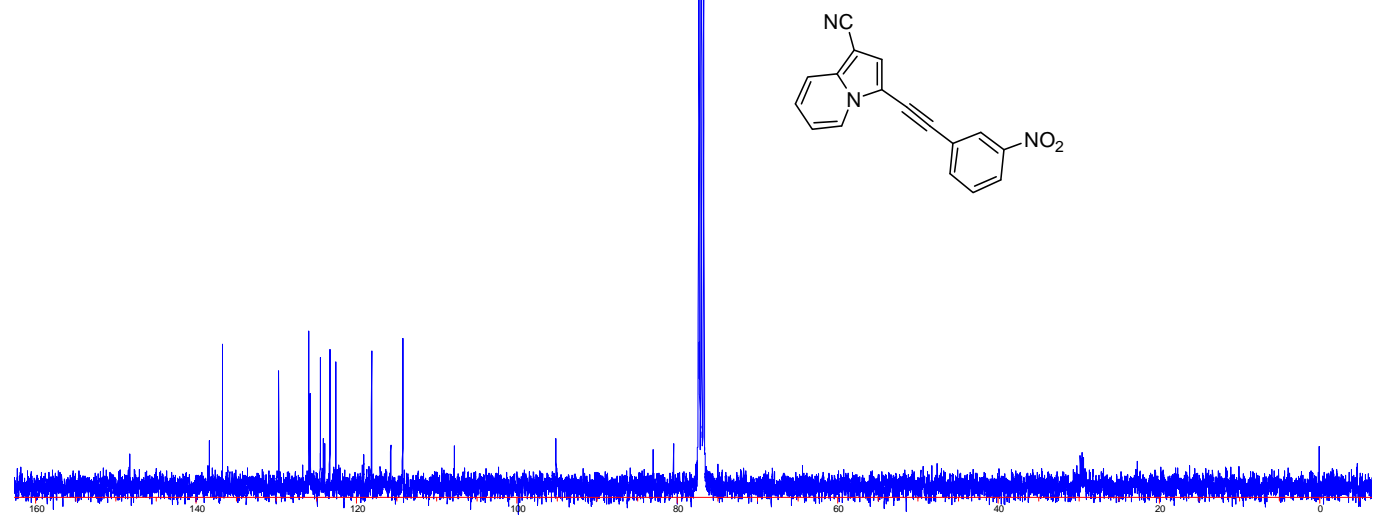


\section{3-(4-Cyano-phenylethynyl)-indolizine-1-carbonitrile ( 4i )}

Yellow solid; mp 173.9-175. ${ }^{\circ} \mathrm{C}$; ${ }^{1} \mathrm{H}$ NMR ( $400 \mathrm{MHz}, \mathrm{CDCl}_{3}$, TMS ) $\delta 8.37$ ( d, $J=8$ $\mathrm{Hz}, 1 \mathrm{H}), 7.71(\mathrm{~d}, 1 \mathrm{H}), 7.62-7.68(\mathrm{~m}, 4 \mathrm{H}), 7.35$ ( s, $1 \mathrm{H}), 7.23$ ( s, $1 \mathrm{H}), 6.99$ ( t, $1 \mathrm{H}) ;{ }^{13} \mathrm{C} \mathrm{NMR}\left(100 \mathrm{MHz}, \mathrm{CDCl}_{3}\right) \delta 175.1,138.4,132.3,131.5,127.1,125.7,124.6$, $122.7,118.8,118.3,118.1,115.7,114.3,111.9,107.9,96.1,83.2,82.3$; IR (neat) $v$ 3100, 2921, 2221, 2198, 1540, 1509, 1487, 1463, 835, $747 \mathrm{~cm}^{-1}$; HRMS (EI) Calcd for $\mathrm{C}_{18} \mathrm{H}_{9} \mathrm{~N}_{3}\left(\mathrm{M}^{+}\right)$267.0796; Found, 267.0794.
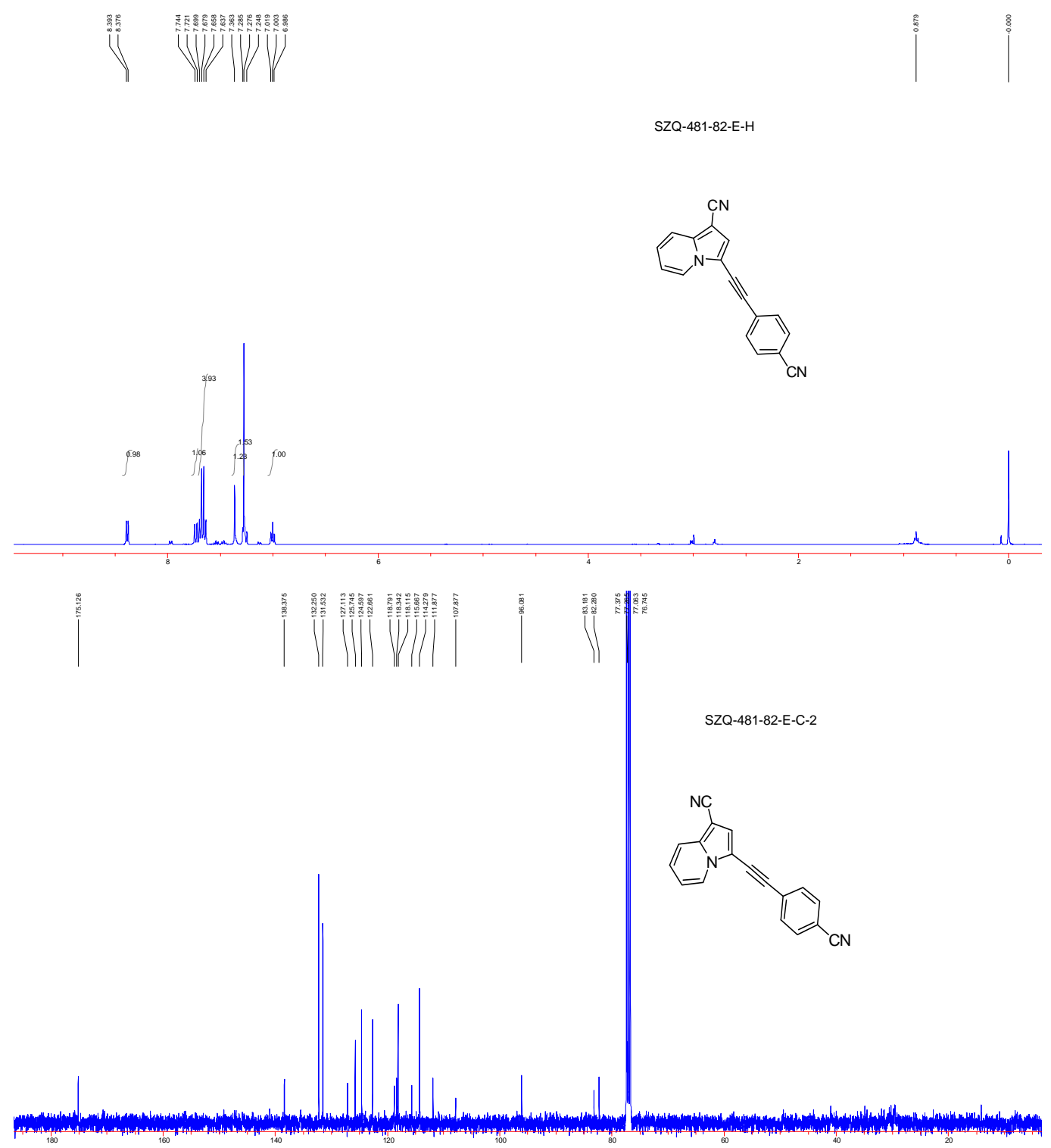


\section{3-((3-methylthiophen-2-yl)ethynyl)indolizine-1-carbonitrile ( $4 \mathbf{j}$ )}

Yellow solid; mp 164.8-166. ${ }^{\circ} \mathrm{C}$; ${ }^{1} \mathrm{H}$ NMR ( $400 \mathrm{MHz}, \mathrm{CDCl}_{3}$, TMS ) $\delta 8.35$ ( d, $J=8$ $\mathrm{Hz}, 1 \mathrm{H}$ ), 7.70 ( d, $J=8 \mathrm{~Hz}, 1 \mathrm{H}$ ), 7.29 ( s, $1 \mathrm{H}$ ), 7.25-7.27 ( m, $2 \mathrm{H}$ ), 7.21 ( m, 1 $\mathrm{H}), 6.93(\mathrm{~m}, 1 \mathrm{H}), 2.41(\mathrm{~s}, 3 \mathrm{H}) ;{ }^{13} \mathrm{C} \mathrm{NMR}\left(100 \mathrm{MHz}, \mathrm{CDCl}_{3}\right) \delta$ 143.7, 138.1, 129.5, 127.2, 125.8, 124.0, 121.7, 118.0, 117.5, 116.1, 113.9, 108.9, 90.0, 83.2, 82.6, 15.3; IR (neat) v 3106, 2921, 2217, 1513, 1463, $747 \mathrm{~cm}^{-1}$; HRMS (EI) Calcd for $\mathrm{C}_{16} \mathrm{H}_{10} \mathrm{~N}_{2} \mathrm{~S}\left(\mathrm{M}^{+}\right)$262.0565; Found, 262.0568.
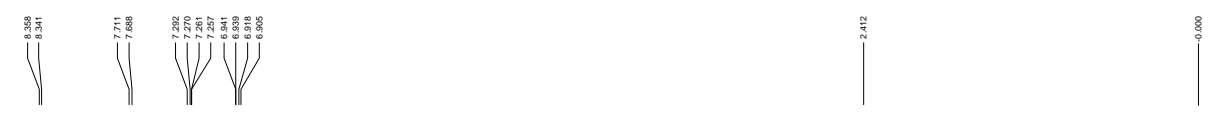

szq-481-75-H
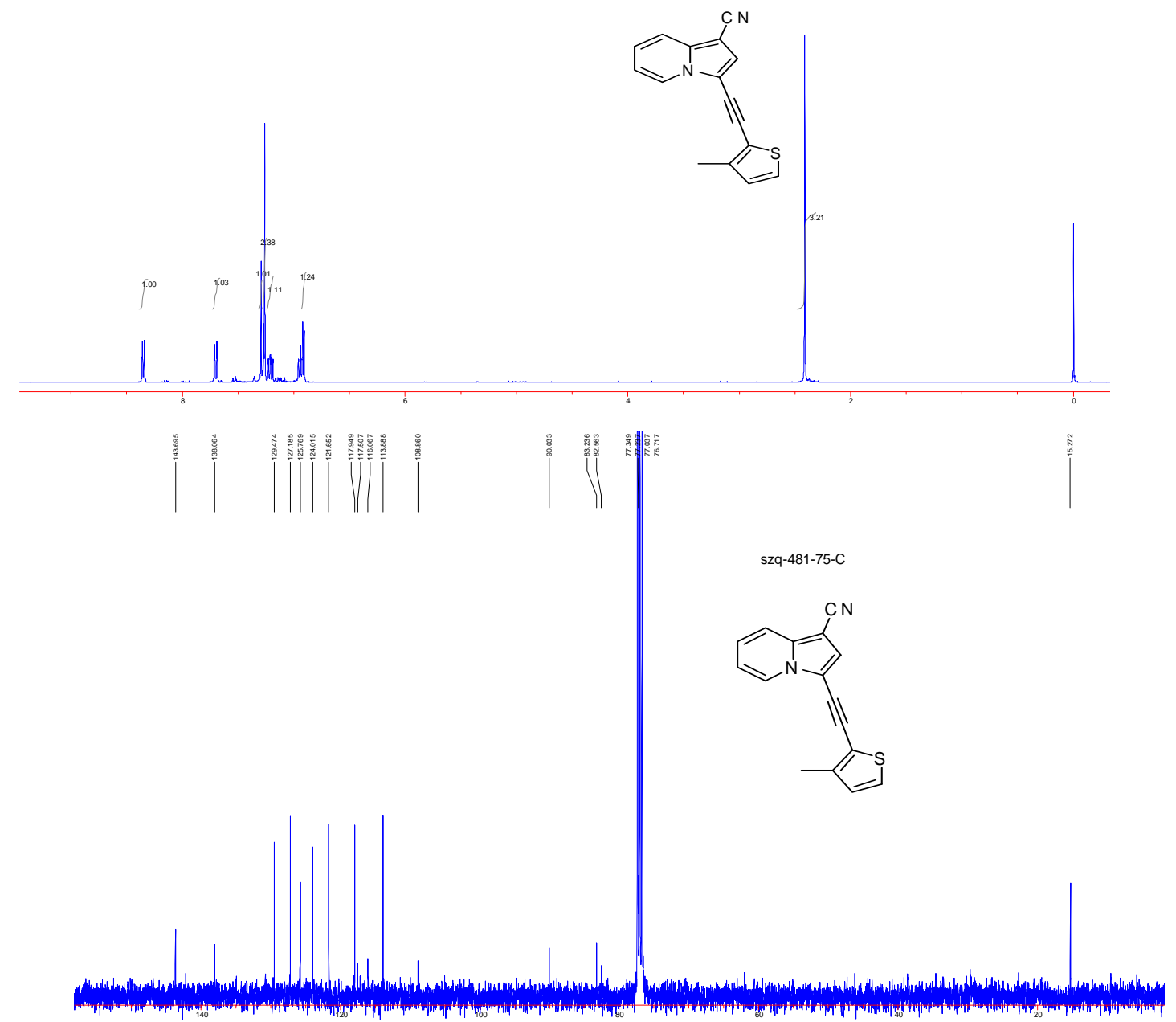
[1] Zhang, L.; Liang, F.; Sun, L.; Hu, Y.; Hu, H. Synthesis 2000, 12, 1733-1737.

[2] Zhao, B. Org. Biomol. Chem. 2012, 10, 7108-7119. 